\title{
Dehydration of an azeotrope of ethanol/water by sodium carboxymethylcellulose membranes cross-linked with organic or inorganic cross-linker
}

\author{
T. Uragami*, D. Wakita, T. Miyata \\ Department of Chemistry and Materials Engineering, High Technology Research Center, Kansai University, Suita, \\ Osaka 564-8680, Japan
}

Received 13 May 2010; accepted in revised form 2 July 2010

\begin{abstract}
To control the swelling of sodium carboxymethylcellulose (CMCNa) membranes, mixtures of CMCNa and glutaraldehyde (GA) and mixtures of CMCNa as an organic component and tetraethoxysilane (TEOS) as an inorganic component were prepared, and $\mathrm{CMCNa} / \mathrm{GA}$ cross-linked membranes and $\mathrm{CMCNa} / \mathrm{TEOS}$ hybrid membranes were formed. In the separation of an ethanol/water azeotrope by pervaporation (PV), the effects of the GA or TEOS content on the water/ethanol selectivity and permeability of these $\mathrm{CMCNa} / \mathrm{GA}$ cross-linked and CMCNa/TEOS hybrid membranes were investigated. Cross-linked and hybrid membranes containing up to $10 \mathrm{wt} \%$ GA or $10 \mathrm{wt} \%$ TEOS exhibited higher water/ethanol selectivity than CMCNa membrane without any cross-linker. This resulted from both increased density and depressed swelling of the membranes by the formation of a cross-linked structure. The relationship between the structure of the $\mathrm{CMCNa} / \mathrm{GA}$ cross-linked membranes and $\mathrm{CMCNa}$ /TEOS hybrid membranes and their permeation and separation characteristics for an ethanol/water azeotrope during PV is discussed in detail.
\end{abstract}

Keywords: biopolymers, biocomposite, polymer membranes, bio-ethanol, dehydration

\section{Introduction}

An aqueous bio-ethanol solution can be produced by fermentation of biomass resources such as dregs of sugar corn, stems of corn and so on, separation and concentration of an aqueous ethanol solution are actively noticed from the viewpoint of a new energy source instead of limited petroleum resource. In general, aqueous alcohol solutions are concentrated by distillation, but an azeotrope $(96.5 \mathrm{wt} \%$ ethanol) cannot be further separated by distillation. In particular, pervaporation (PV) is a promising technique for separation of azeotropes $[1,2]$ such as an aqueous solution of $96.5 \mathrm{wt} \%$ ethanol, and close boiling point mixtures $[3,4]$ through polymer membranes. In PV separation, membrane performance is highly dependent upon the physical and chemical structures of the membrane. Therefore, carefully designing the membrane structure is required to develop membranes with high selectivity and permeability. We have studied several kinds of cross-linked polymer membranes and organicinorganic hybrid membranes to separate aqueous ethanol solutions during PV. For example, chitosan membranes cross-linked with glutaraldehyde [5], quaternized chitosan membranes cross-linked with glutaraldehyde [6], ethylenglycoldiglycidylether [7] and poly(ethylene oxydiglycolic acid) [8], poly(vinyl alcohol) and tetraethoxysilane (TEOS) [9], poly(vinyl alcohol-co-acrylic acid) and TEOS [10], and quaternized chitosan and TEOS [11] hybrid membranes prepared by sol-gel reaction all showed high water/ethanol selectivity for an

*Corresponding author, e-mail: uragami@kansai-u.ac.jp

(C) BME-PT 
azeotrope of ethanol/water because their membrane swelling could be effectively controlled by crosslinking or hybridization.

In this study, sodium carboxymethylcellulose $(\mathrm{CMCNa})$ was selected as a membrane material because $\mathrm{CMCNa}$ can be easily formed into fine membrane. In general, $\mathrm{CMCNa}$ has been applied as sizing in paper fabrication, as emulsion stabilizer, in water-borne paints and so on. We prepared sodium carboxymethylcellulose $(\mathrm{CMCNa}) \mathrm{mem}$ branes cross-linked with glutaraldehyde (GA) and novel organic-inorganic hybrid membranes by the sol-gel reaction between $\mathrm{CMCNa}$ as an organic component and tetraethoxysilane (TEOS) as an inorganic component in order to reduce the swelling of the $\mathrm{CMCNa}$ membranes. Furthermore, the permeation and separation characteristics of an azeotrope of ethanol/water through the $\mathrm{CMCNa} / \mathrm{GA}$ crosslinkd membranes and the CMCNa/TEOS hybrid membranes during PV are discussed as a function of the GA or TEOS content. Recently, organicinorganic hybrid materials are recognized as significant composite materials with both the functionality of an organic compound and with the strength of an inorganic compound. Therefore, in this study we call $\mathrm{CMCNa}$ /TEOS cross-linked membranes as $\mathrm{CMCNa}$ /TEOS hybrid membranes.

\section{Materials and methods}

\subsection{Materials}

Sodium carboxymethylcellulose (CMCNa), whose degree of sodium carboxymethylation is 0.91 purchased from Tokyo Kasei Industry Co. Ltd., Japan. GA and trifluoroacetic acid anhydride were supplied by Wako Chemical Industry Co. Ltd., Japan. TEOS was supplied by Shinetsu Chemical Industry Co. Ltd., Japan. All other reagents and solvents used in this study were supplied by commercial sources.

\subsection{Preparation of $\mathrm{CMCNa}$ membrane, $\mathrm{CMCNa}$ /GA cross-linked membranes and $\mathrm{CMCNa}$ /TEOS hybrid membranes}

The CMCNa membranes were prepared by pouring $3 \mathrm{wt} \% \mathrm{CMCNa}$ in $0.01 \mathrm{M} \mathrm{HCl}$ onto a glass plate treated with silicon, allowing water to evaporate at $50^{\circ} \mathrm{C}$ for $10 \mathrm{~h}$ in an oven, and then drying under reduced pressure. The $\mathrm{CMCNa} / \mathrm{GA}$ cross-linked and $\mathrm{CMCNa}$ /TEOS hybrid membranes were prepared by pouring $3 \mathrm{wt} \% \mathrm{CMCNa}$ in $0.01 \mathrm{M} \mathrm{HCl}$ with a desired amount of GA as an organic crosslinker or a desired amount of TEOS as an inorganic cross-linker onto a glass plate treated with silicon, allowing water to evaporate at $50^{\circ} \mathrm{C}$ for $10 \mathrm{~h}$ in an oven, and then drying under reduced pressure. The resulting $\mathrm{CMCNa}$ membranes, $\mathrm{CMCNa} / \mathrm{GA}$ crosslinked membranes and $\mathrm{CMCNa}$ /TEOS hybrid membranes were kept in a desiccator at room temperature in vacuo.

\subsection{PV apparatus and measurements}

The PV cell and PV apparatus have been described in a previous paper [12]. The effective membrane area was about $13.8 \mathrm{~cm}^{2}$. The PV experiments were carried out under the following conditions: permeation temperature, $40^{\circ} \mathrm{C}$; and pressure of the permeate side, $1.33 \mathrm{~Pa}$. The feed solution, an aqueous solution of $96.5 \mathrm{wt} \%$ ethanol, was circulated between the PV cell and the feed tank to maintain a constant concentration of feed solution in the PV cell. The permeation rate $\left[\mathrm{kg} / \mathrm{m}^{2} \cdot \mathrm{h}\right]$ for an azeotrope of ethanol/water during PV was determined from the weight $[\mathrm{kg}]$ of permeate collected in a cold trap, the permeation time $[\mathrm{h}]$ and the effective membrane area $\left[\mathrm{m}^{2}\right]$. The results of the permeation for an azeotrope of ethanol/water by PV were reproducible, and the errors inherent in these permeation measurements ranged within a few percent of the permeation rates through the membranes. The permeation results in this study were determined after steady-state flux.

The ethanol concentration in the feed and permeate was determined by means of a gas chromatograph (Shimadzu GC-14A, Japan) using a flame ionization detector (FID) and a capillary column (Shimadzu Co. Ltd: PorapacQ, Japan) heated to $180^{\circ} \mathrm{C}$. The separation factor, $\alpha_{\text {sep. } \mathrm{H}_{2} \mathrm{O} / \mathrm{EtOH} \text {, was calculated }}$ from Equation (1):

$$
\alpha_{\text {sep. } \mathrm{H}_{2} \mathrm{O} / \mathrm{EtOH}}=\frac{P_{\mathrm{H}_{2} \mathrm{O}} / P_{\mathrm{EtOH}}}{F_{\mathrm{H}_{2} \mathrm{O}} / F_{\mathrm{EtOH}}}
$$

where $F_{\mathrm{EtOH}}$ and $F_{\mathrm{H}_{2} \mathrm{O}}$ are the weight fractions of ethanol and water in the feed solution, and $P_{\mathrm{EtOH}}$ and $P_{\mathrm{H}_{2} \mathrm{O}}$ are those in the permeate, respectively. 


\subsection{Composition of solution absorbed into CMCNa/GA cross-linked membranes and $\mathrm{CMCNa}$ /TEOS hybrid membranes}

The $\mathrm{CMCNa} / \mathrm{GA}$ cross-linked and $\mathrm{CMCNa} / \mathrm{TEOS}$ hybrid membranes were dried completely under reduced pressure at room temperature and weighed. The dried membranes were immersed into an azeotropic mixture of ethanol/water in a sealed vessel at $40^{\circ} \mathrm{C}$ until equilibrium was reached. A large amount of the swollen $\mathrm{CMCNa} / \mathrm{GA}$ cross-linked and $\mathrm{CMCNa}$ /TEOS hybrid membranes was placed in a container as reported in a previous paper [13]. The system was evacuated, and the container with the swollen membranes was then heated. The solution sorbed into the swollen membranes was completely desorbed under reduced pressure, and was collected in a U-tube cooled with liquid nitrogen. The composition of the solutions sorbed into the $\mathrm{CMCNa} / \mathrm{GA}$ cross-linked and $\mathrm{CMCNa} / \mathrm{TEOS}$ hybrid membranes was then determined by measuring the ethanol concentration in the collected solution by gas chromatography (Shimadzu GC-14A). The ethanol/water composition in the $\mathrm{CMCNa} / \mathrm{GA}$ cross-linked and $\mathrm{CMCNa}$ /TEOS hybrid membranes versus that in the feed solution yielded the sorption selectivity, $\alpha_{\text {sorp. } \mathrm{H}_{2} \mathrm{O} / \mathrm{EtOH}}$, as expressed in Equation (2):

$\alpha_{\text {sorp. } \mathrm{H}_{2} \mathrm{O} / \mathrm{EtOH}}=\frac{M_{\mathrm{H}_{2} \mathrm{O}} / M_{\mathrm{EtOH}}}{F_{\mathrm{H}_{2} \mathrm{O}} / F_{\mathrm{EtOH}}}$

where $F_{\mathrm{H}_{2} \mathrm{O}}$ and $F_{\mathrm{EtOH}}$ are the weight fractions of water and ethanol in the feed solution, and $M_{\mathrm{H}_{2} \mathrm{O}}$ and $M_{\mathrm{EtOH}}$ are those sorbed into the membrane, respectively.

\subsection{Degree of swelling}

The $\mathrm{CMCNa}$ and $\mathrm{CMCNa} / \mathrm{GA}$ cross-linked and $\mathrm{CMCNa}$ /TEOS hybrid membranes were completely dried under reduced pressure at $40^{\circ} \mathrm{C}$ and weighed. These dried membranes were immersed in an ethanol/water azeotrope ( $96.5 \mathrm{wt} \%$ ethanol) in a sealed vessel at $40^{\circ} \mathrm{C}$ until equilibrium was reached. The membranes were removed from the vessel, wiped quickly with filter paper, and weighed. The degree of swelling of the membrane (DS) was determined from Equation (3):

$$
D S=\frac{W_{s}}{W_{d}}
$$

where $W_{d}$ and $W_{s}$ are the weights of the dried membrane and swollen membrane in the feed, respectively.

\subsection{Measurement of the contact angle}

The contact angle for methylene iodide on the surface of the $\mathrm{CMCNa} / \mathrm{GA}$ cross-linked and $\mathrm{CMCNa} /$ TEOS hybrid membranes was measured by a contact angle meter (Dropmaster Kyouwa Sci. Co. Ltd.) at $25^{\circ} \mathrm{C}$. The contact angle, $\theta$, was calculated from Equation (4) [14]:

$\theta=\cos ^{-1}\left(\frac{\cos \theta_{a}+\cos \theta_{r}}{2}\right)$

where $\theta_{a}$ and $\theta_{r}$ are the advancing contact angle and the receding contact angle, respectively.

\subsection{X-ray photoelectron spectroscopy (XPS) measurements}

To characterize the surfaces of the $\mathrm{CMCNa}$ membrane, the $\mathrm{CMCNa} / \mathrm{GA}$ cross-linked and $\mathrm{CMCNa} /$ TEOS hybrid membranes, the hydroxyl groups of their surfaces were fluorinated by trifluroacetic acid anhydride as shown by Equation (5):
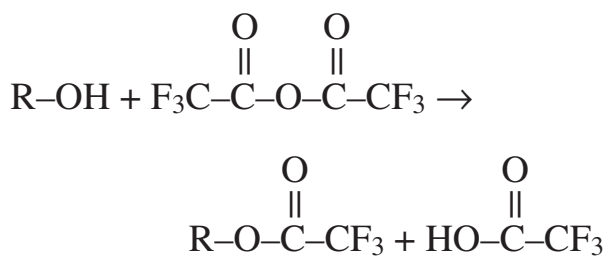

The fluorination was carried out as follows. Specimens of these membranes $(5 \mathrm{~mm} \times 5 \mathrm{~mm})$ were immersed in a mixture of benzene $(20 \mathrm{ml})$, pyridine $(4 \mathrm{ml})$ and trifluoroacetic acid anhydride $(4 \mathrm{ml})$ for $1.5 \mathrm{~h}$ at room temperature, and the immersed specimens were washed with benzene repeatedly. Next, they were placed in a Soxhlet extractor with diethylether for $12 \mathrm{~h}$ to completely remove the trifluroacetic acid anhydride and trifluroacetic acid, and then dried in a desiccator under reduced pressure at room temperature. 
The fluorinated surfaces of these membranes were characterized with an X-ray photoelectron spectroscope (XPS) (Shimadzu, ESCA-3400, Japan). The typical operating conditions were as follows: $\mathrm{CuK}_{\alpha}$ radiation of $15 \mathrm{kV}$ and $10 \mathrm{~mA}$. The pressure in the instrument chamber was less than $1.0 \cdot 10^{-6} \mathrm{~Pa}$. No radiation damage was observed during the data collection time.

The atomic compositions on the surface of the $\mathrm{CMCNa} / \mathrm{GA}$ cross-linked and $\mathrm{CMCNa} / \mathrm{TEOS}$ hybrid membranes were determined by Equations (6) and (7):

$\mathrm{CMCNa} /(\mathrm{CMCNa}+\mathrm{TEOS})=\mathrm{F} /(\mathrm{F}+\mathrm{Si})$

$\mathrm{CMCNa} /(\mathrm{CMCNa}+\mathrm{GA})=\mathrm{F} /(\mathrm{F}+\mathrm{O})$

where $\mathrm{F}, \mathrm{Si}$ and $\mathrm{O}$ are the fractions of each element on the surface of the $\mathrm{CMCNa} / \mathrm{GA}$ membranes and $\mathrm{CMCNa}$ /TEOS membranes.

\subsection{Transmission electron micrographs (TEM)}

The $\mathrm{CMCNa}$ /GA cross-linked and $\mathrm{CMCNa}$ /TEOS hybrid membranes were embedded in epoxy resin and sliced into thin films (thickness $\approx 50 \mathrm{~nm}$ ) with a microtome (Leica; Reichert Ultracut E). The structure of the membranes was observed by a transmission electron microscope (TEM) (JEOL JEM$120 \mathrm{~L}$, Japan) at an accelerating voltage of $80 \mathrm{kV}$.

\section{Results and discussion}

\subsection{Effect of the cross-linker content on the permeation and separation characteristics}

Figure 1 shows the effects of the cross-linker content on the permeation rate and separation factor for an azeotrope of ethanol/water through the $\mathrm{CMCNa}$ / GA cross-linked and CMCNa/TEOS hybrid membranes during PV. As can be seen from Figure 1, the separation factors of all $\mathrm{CMCNa} / \mathrm{GA}$ crosslinked and $\mathrm{CMCNa} / \mathrm{TEOS}$ hybrid membranes were higher than those of the neat CMCNa membrane. These results support the contention that all membranes had water/ethanol selectivity. The separation factors of the CMCNa/TEOS hybrid membranes were higher than those of $\mathrm{CMCNa} / \mathrm{GA}$ cross-linked membranes. Furthermore, in both $\mathrm{CMCNa} / \mathrm{GA}$ cross-linked and CMCNa/TEOS
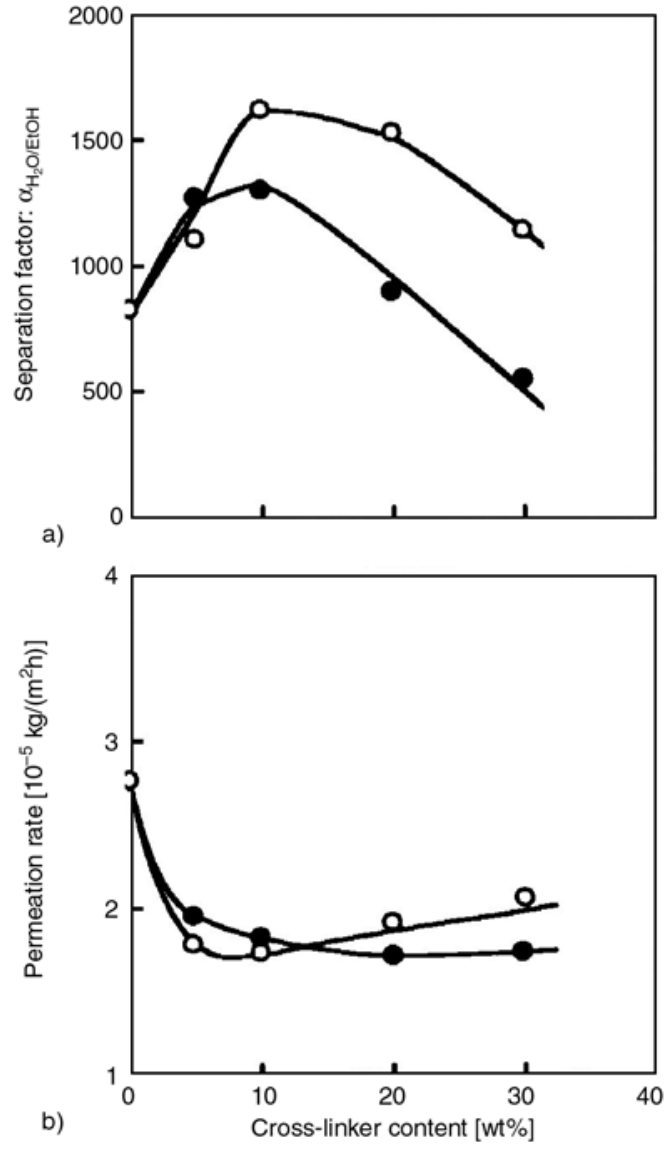

Figure 1. Effects of the GA or TEOS content on the permeation rate and separation factor for an azeotrope of ethanol/water through $\mathrm{CMCNa} / \mathrm{GA}$ cross-linked membranes $(\bullet)$ or $\mathrm{CMCNa} / \mathrm{TEOS}$ hybrid membranes (o) during $\mathrm{PV}$ at $40^{\circ} \mathrm{C}$

hybrid membranes, the membranes cross-linked with $10 \mathrm{wt} \%$ of cross-linker showed the highest separation factors. On the other hand, one can understand that the permeation rates of both $\mathrm{CMCNa} /$ TEOS hybrid and $\mathrm{CMCNa} / \mathrm{GA}$ cross-linked membranes decreased until $10 \mathrm{wt} \%$ of cross-linker content, but above this content, the dependency of permeation rate on the cross-linker content significantly differ. Thus, the permeation rate in the $\mathrm{CMCNa}$ /TEOS membrane increased but that in the $\mathrm{CMCNa} / \mathrm{GA}$ membrane decreased slightly.

\subsection{Chemical structure of $\mathrm{CMCNa} / \mathrm{GA}$ cross- linked membranes and $\mathrm{CMCNa}$ /TEOS hybrid membranes}

In general, the permeation and separation characteristics of these membranes are significantly governed by the chemical and physical structure of the membrane. Thus, it is known that the chemical 
structure influences the solubility of the permeants into the membrane and the physical structure affects the diffusivity of the permeants through the membrane. Therefore, we analyzed the chemical structure of the membranes by the measuring the composition of solution in the membrane, the contact angle of the membrane surface and XPS, and the physical structure by the measuring the membrane density, and the degree of swelling of the membranes, and by TEM observation to elucidate their permeation and separation characteristics, as shown in Figure 1.

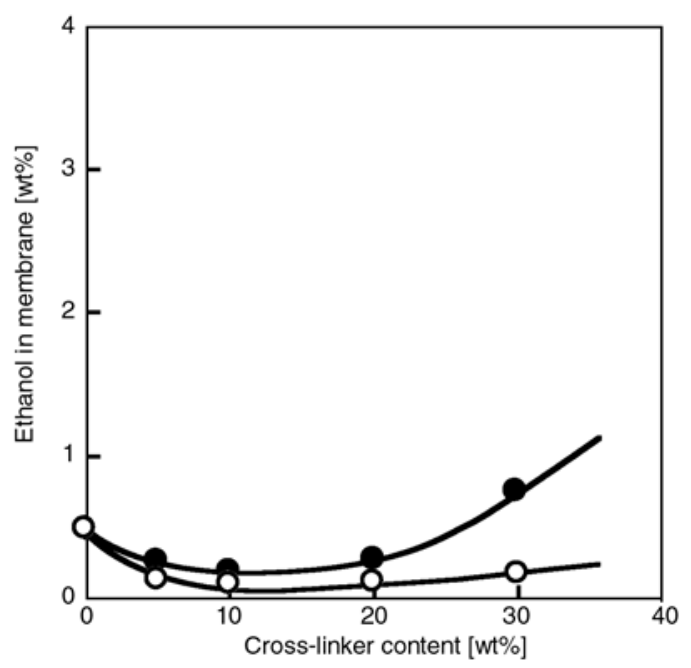

Figure 2. Effect of the cross-linker content on the ethanol concentration in the $\mathrm{CMCNa} / \mathrm{GA}$ cross-linked membranes $(\bullet)$ and $\mathrm{CMCNa}$ /TEOS hybrid membranes (o) immersed in an aqueous solution of $96.5 \mathrm{wt} \%$ ethanol at $40^{\circ} \mathrm{C}$

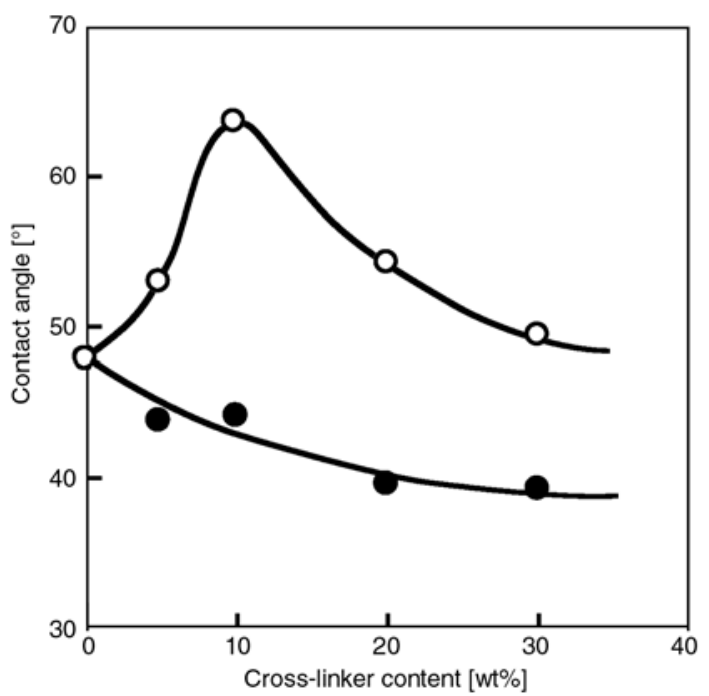

Figure 3. Contact angle for methylene iodide on the surface of $\mathrm{CMCNa} / \mathrm{GA}$ cross-linked membranes (•) and $\mathrm{CMCNa}$ /TEOS hybrid membranes (o) as a function of the cross-linker content
In Figures 2 and 3, the ethanol concentration sorbed into the membrane immersed in an azeotrope of ethanol/water and the contact angle of the membrane surface for methylene iodide as a function of the cross-linker content are shown. As can be seen from Figure 2, the ethanol concentration sorbed into the membrane for both the $\mathrm{CMCNa} / \mathrm{GA}$ crosslinked and $\mathrm{CMCNa}$ /TEOS hybrid membranes decreased slightly with increasing content up to $10 \mathrm{wt} \%$ of GA or TEOS, whereas the ethanol concentration in the CMCNa/TEOS membrane increased faintly, and the ethanol concentration in the CMCNA/GA membrane increased more than $10 \mathrm{wt} \%$ of cross-linker content.

On the other hand, the sorbed ethanol concentration for the $\mathrm{CMCNa}$ /TEOS hybrid membranes was lower than that of the $\mathrm{CMCNa} / \mathrm{GA}$ cross-linked membranes over the whole range cross-linker contents. The higher separation factors for the $\mathrm{CMCNa} /$ TEOS hybrid membranes in Figure 1 are dependent on this result.

In Figure 3, the increase or decrease in the contact angle for methylene iodide, which is a hydrophobic solvent, suggests that the membrane surface became more hydrophilic or hydrophobic with an increase in the cross-linker content, respectively. The contact angles of the CMCNa/TEOS hybrid membranes were higher than those of the $\mathrm{CMCNa} / \mathrm{GA}$ cross-linked membranes. This result suggests that the former membranes are more hydrophilic than the latter. In the $\mathrm{CMCNa}$ /TEOS hybrid membranes, the contact angle at $10 \mathrm{wt} \%$ of TEOS showed a maximum, and all contact angles were higher than that of the CMCNa membrane. These results suggest that the $\mathrm{CMCNa}$ /TEOS hybrid membranes are more hydrophilic than $\mathrm{CMCNa}$ membranes without TEOS. The contact angles of the $\mathrm{CMCNa} / \mathrm{GA}$ cross-linked membranes decreased with an increasing GA content, and were lower than that of the membrane without GA. This suggests that the $\mathrm{CMCNa} / \mathrm{GA}$ cross-linked membranes are more hydrophobic than membranes without GA. The higher hydrophilicity of the $\mathrm{CMCNa}$ /TEOS hybrid membranes could be attributed to the fact that some silanol groups from the four silanol groups formed from TEOS form the hydrogen and covalent bonds by reacting with the hydroxyl groups in $\mathrm{CMCNa}$ and remained silanol groups from TEOS can be introduced into the $\mathrm{CMCNa} / \mathrm{TEOS}$ membrane, and consequently hydrophilic silanol groups in $\mathrm{CMCNa} /$ 
TEOS membrane increase, and thus the higher hydrophobicity of the $\mathrm{CMCNa} / \mathrm{GA}$ cross-linked membranes could be due to the fact that the hydroxyl groups in the CMCNa molecule can be consumed by the condensation reaction between the aldehyde group in the GA molecule and the hydroxyl group in the $\mathrm{CMCNa}$ molecule.

\subsection{XPS measurements of the membranes}

To determine the chemical structure of the surface of the $\mathrm{CMCNa} / \mathrm{GA}$ cross-linked and $\mathrm{CMCNa}$ / TEOS hybrid membranes in more detail, the hydroxyl groups on the surface of cross-linked and hybrid membranes were labeled by trifluoroacetic acid anhydride as per Equation (5).

In Figures 4 and 5, the XPS spectra of the fluorinated surfaces of $\mathrm{CMCNa} / \mathrm{GA}$ cross-linked and $\mathrm{CMCNa}$ /TEOS hybrid membranes are shown, respectively. In Figure 4, with increasing GA content, the peak intensity assigned to the $\mathrm{F}$ atom at $690 \mathrm{eV}$ decreased, whereas the peak intensity due to the $\mathrm{O}$ atom at about $532 \mathrm{eV}$ increased. On the other hand, as can be seen in Figure 5, the peak intensity assigned to the $\mathrm{Si}$ atom at $105 \mathrm{eV}$ increased and the $\mathrm{F}$ atom at $690 \mathrm{eV}$ decreased slightly upon introducing TEOS into the CMCNa membrane. In

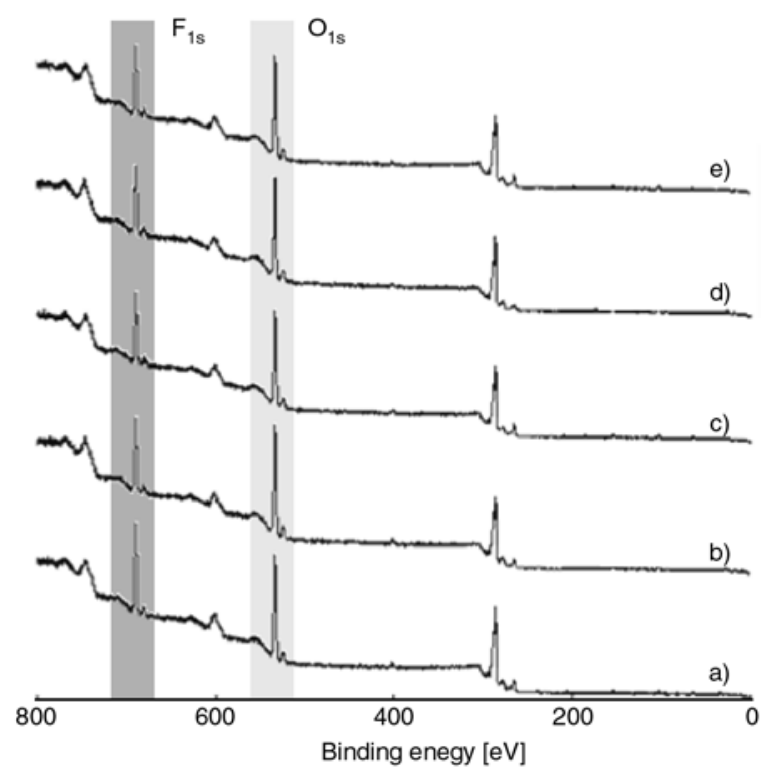

Figure 4. XPS spectra of (a) the CMCNa membrane, (b) a GA content of $5 \mathrm{wt} \%$, (c) a GA content of $10 \mathrm{wt} \%$, (d) a GA content of $20 \mathrm{wt} \%$ and (e) a GA content of $30 \mathrm{wt} \%$ in the $\mathrm{CMCNa} / \mathrm{GA}$ crosslinked membranes. The surfaces of these membranes were treated with trifluoroacetic acid anhydride

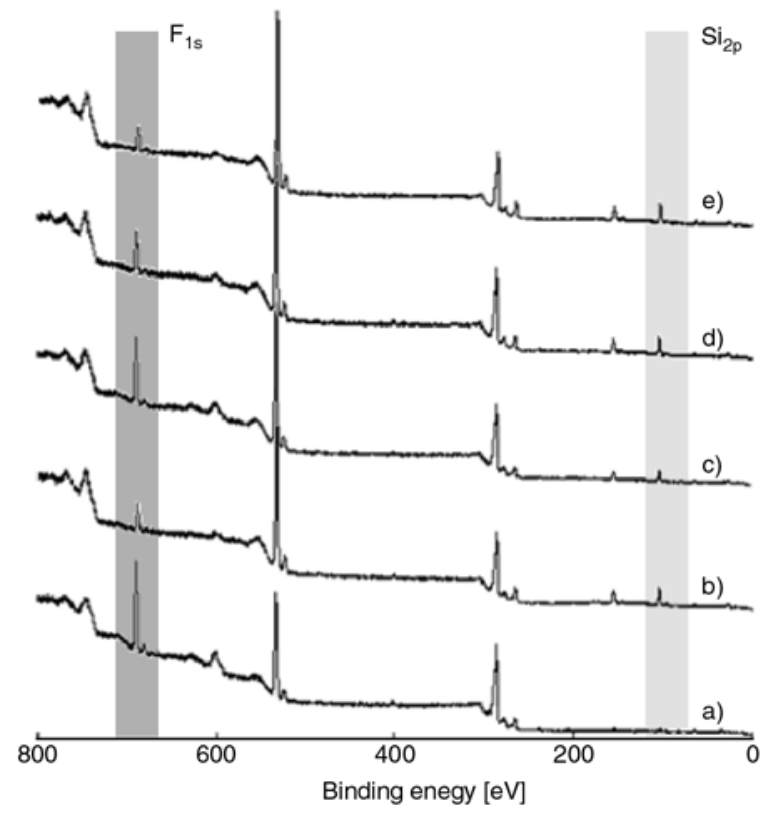

Figure 5. XPS spectra of (a) the CMCNa membrane, (b) a TEOS content of $5 \mathrm{wt} \%$, (c) a TEOS content of $10 \mathrm{wt} \%$, (d) a TEOS content of $20 \mathrm{wt} \%$ and (e) a TEOS content of $30 \mathrm{wt} \%$ in the CMCNa/TEOS hybrid membranes. The surfaces of these membranes were treated with trifluoroacetic acid anhydride

the spectrum (c) of the CMCNa/TEOS hybrid membrane with a TEOS content of $10 \mathrm{wt} \%$, however, the peak intensity of the $\mathrm{F}$ atom increased but that of the Si atom decreased. Above $10 \mathrm{wt} \%$ of TEOS content, the peak intensity at the $\mathrm{F}$ atom decreased and that of the $\mathrm{Si}$ atom increased. The results from Figures 4 and 5 support the results of the contact angle measurements in Figure 3. Thus, it is found that the networks of $\mathrm{CMCNa} / \mathrm{GA}$ cross-linked and $\mathrm{CMCNa}$ /TEOS hybrid membranes and the amount of the hydroxyl and silanol groups on the surface of these membranes were significantly influenced by the GA or TEOS content.

In Figure 6, the effects of the cross-linker content on the atomic compositions on the surface of the $\mathrm{CMCNa} / \mathrm{GA}$ cross-linked and $\mathrm{CMCNa} / \mathrm{TESO}$ hybrid membranes are shown, respectively. With increasing GA content, the amount of $\mathrm{F}$ atoms decreased because of the decrease in the hydroxyl group based on the condensation reaction between the hydroxyl groups in the CMCNa molecule and the aldehyde groups in the GA molecule. In Figure 6, the introduction of a small amount of TEOS into the $\mathrm{CMCNa}$ accelerated the reactions between the hydroxyl groups and the silanol groups formed due to the hydrolysis of TEOS and the amount of 


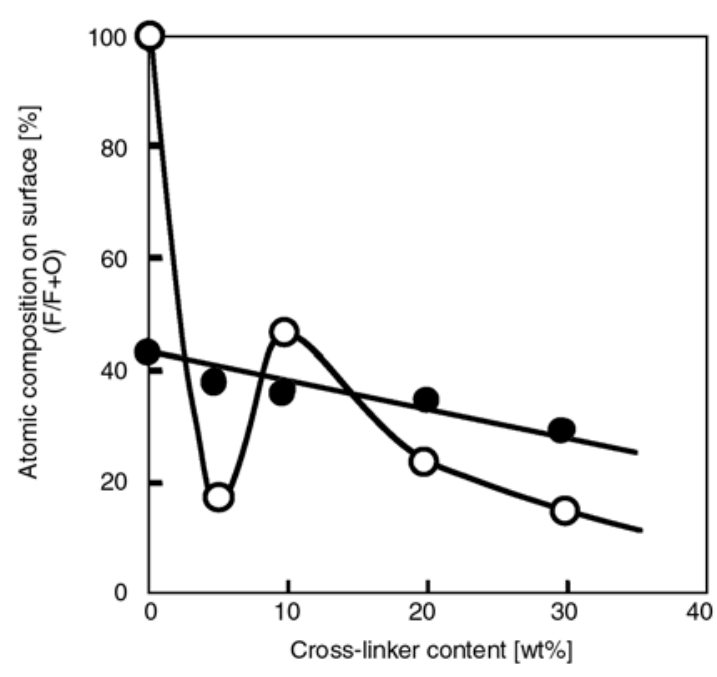

Figure 6. Effect of the cross-linker content on the atomic composition on the surface of $\mathrm{CMCNa} / \mathrm{GA}$ cross-linked membranes $(\bullet)$ and $\mathrm{CMCNa} / \mathrm{TEOS}$ hybrid membranes (o)

hydroxyl groups in the CMCNa molecule decreased and consequently the amount of $\mathrm{F}$ atoms decreased. At $10 \mathrm{wt} \%$ of TEOS content, the amount of $\mathrm{F}$ atoms increased due to the increase in the silanol group during the sol-gel process. Above $10 \mathrm{wt} \%$ of TEOS content, since the number of hydroxyl groups were decreased by excess condensation reactions, the amount of $\mathrm{F}$ atom decreased. These findings also support those observed in the contact angle measurements.

\subsection{Structure of CMCNa/TEOS hybrid membranes}

Based on the above results, the structure of $\mathrm{CMCNa}$ /TEOS hybrid membranes is illustrated in Figure 7, showing the process polycondensation reaction of TEOS [15]. In the first step of the process of preparing the $\mathrm{CMCNa}$ /TEOS hybrid membranes, the silanol groups were formed by hydrolyzing TEOS in the presence of an acid catalyst (A). The resulting silanol groups yielded siloxane bonds due to the dehydration or dealcoholysis

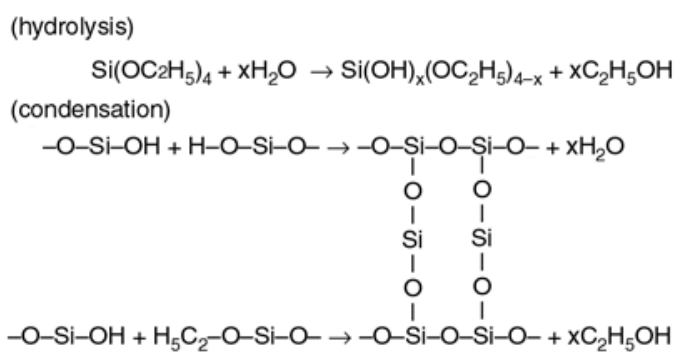

Figure 7. Hydrolysis and condensation scheme for TEOS

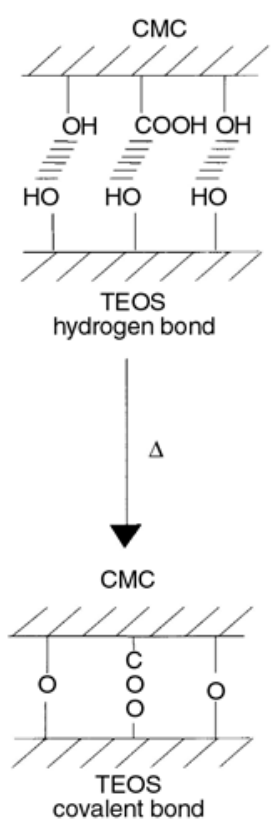

Figure 8. Illustration of the interaction between $\mathrm{CMCNa}$ and TEOS

reactions with other silanol groups or ethoxy groups during the membrane drying process (B). These reactions led to cohesive bodies between siloxane groups in the membrane. Since the siloxane groups were dispersed in the membrane, the silanol groups in the siloxane and the hydroxyl groups in $\mathrm{CMCNa}$ formed hydrogen and covalent bonds, which are the cross-linking points, as illustrated in Figure 8.

However, the dehydration and dealcoholysis reactions occurred predominantly rather than the formation of hydrogen and covalent bonds. Thus, a rough cohesive structure of TEOS was formed, and hence a membrane with a more open structure was produced, leading to a decrease in the water/ethanol selectivity.

\subsection{Physical structure of $\mathrm{CMCNa} / \mathrm{GA}$ cross-linked and CMCNa/TEOS hybrid membranes}

In Figure 9, the degree of swelling of the $\mathrm{CMCNa} /$ GA cross-linked and CMCNa/TEOS hybrid membranes is shown as a function of the GA or TEOS content. The degree of swelling of both membranes decreased with an increase in the GA or TEOS content. The swelling of the $\mathrm{CMCNa}$ /TEOS hybrid membranes was smaller than that of the $\mathrm{CMCNa} /$ GA cross-linked membranes. These results can be supported by the results of the membrane density. 


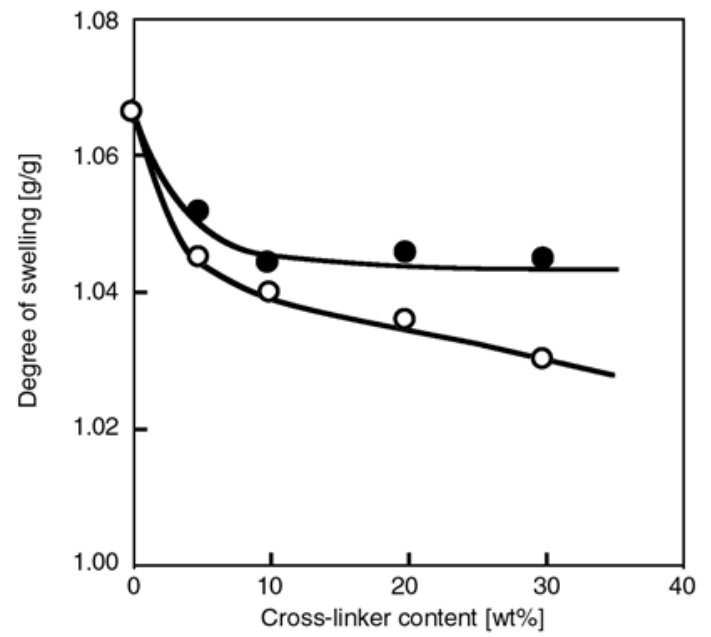

Figure 9. Effect of the cross-linker content on the degree of swelling of $\mathrm{CMCNa} / \mathrm{GA}$ cross-linked membranes $(\bullet)$ and $\mathrm{CMCNa}$ /TEOS hybrid membranes (o)

\subsection{Permselectivity mechanism of cross- linked and hybrid membranes}

In the solution-diffusion model $[16,17]$ differences in the solubility of the permeants in the membranes and differences in the diffusivity of the permeants in the polymer membranes are very significantly related to their permselectivity [18]. It is very important to determine the sorption selectivity and diffusion selectivity to elucidate the separation mechanism of an azeotrope of ethanol/water through $\mathrm{CMCNa} / \mathrm{GA}$ cross-linked and $\mathrm{MCNa} / \mathrm{TEOS}$ hybrid membranes. Thus, in order to discuss the water/ ethanol selectivity of an azeotrope of ethanol/water through $\mathrm{CMCNa} / \mathrm{GA}$ cross-linked and $\mathrm{CMCNa} /$ TEOS hybrid membranes from the viewpoint of the solution-diffusion mechanism, both the sorption selectivity and the diffusion selectivity must be

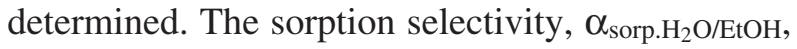
was determined from Equation (2), whereas the diffusion selectivity, $\alpha_{\text {diff. } \mathrm{H}_{2} \mathrm{O} / \mathrm{EtOH}}$, could be calculated from Equation (8) using Equations (1) and (2):

$\alpha_{\text {diff. } \mathrm{H}_{2} \mathrm{O} / \mathrm{EtOH}}=\frac{\alpha_{\text {sep. } \mathrm{H}_{2} \mathrm{O} / \mathrm{EtOH}}}{\alpha_{\text {sorp. } \mathrm{H}_{2} \mathrm{O} / \mathrm{EtOH}}}$

In Figure 10, the separation factor, the sorption selectivity and the diffusion selectivity for an azeotrope of ethanol/water through $\mathrm{CMCNa} / \mathrm{GA}$ cross-linked or $\mathrm{CMCNa} / \mathrm{TEOS}$ hybrid membranes are shown as a function of the GA or TEOS content. The sorption selectivities of all membranes
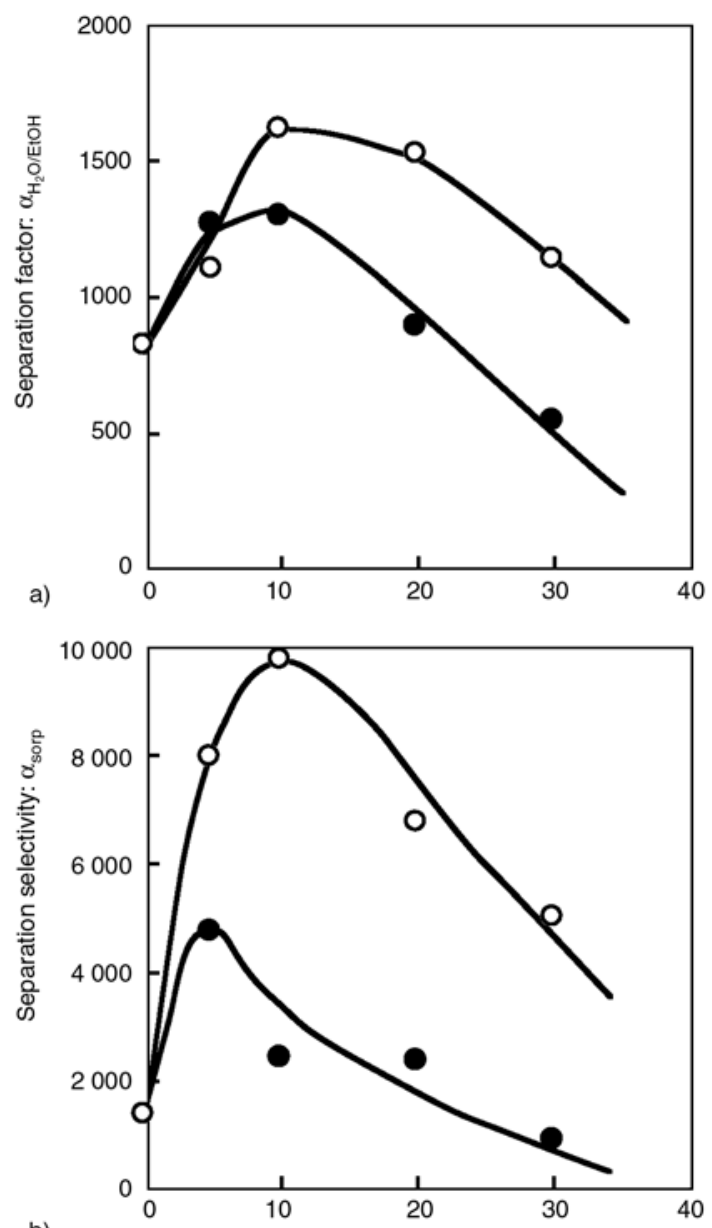

b)

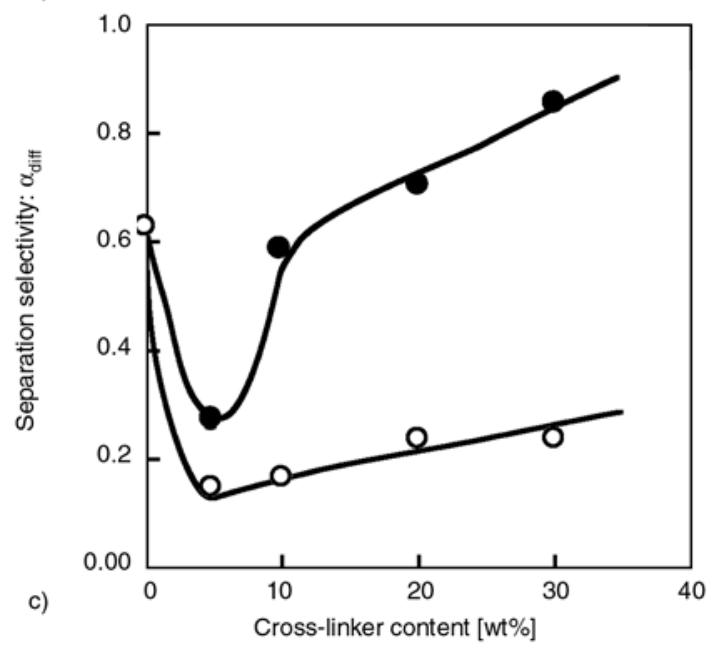

Figure 10. Effects of the cross-linker content on the separation factor, the sorption selectivity and the diffusin selectivity for an azeotrope of ethanol/ water through $\mathrm{CMCNa} / \mathrm{GA}$ cross-linked membranes $(\bullet)$ and $\mathrm{CMCNa} / \mathrm{TEOS}$ hybrid membranes (o) during PV at $40^{\circ} \mathrm{C}$

with various GA or TEOS contents were greater than their diffusion selectivities. This observation suggests that the dehydration process from an aqueous solution of $96.5 \mathrm{wt} \%$ ethanol using $\mathrm{CMCNa} / \mathrm{GA}$ 
cross-linked and $\mathrm{CMCNa}$ /TEOS hybrid membranes is mainly governed by the sorption process. The fact that water was preferentially absorbed into these membranes rather than ethanol also supports this conclusion. Furthermore, the sorption selectivities of the $\mathrm{CMCNa} / \mathrm{TEOS}$ hybrid membranes were higher than those of the $\mathrm{CMCNa} / \mathrm{GA}$ cross-linked membranes. These results are supported by the composition absorbed into the membrane as shown in Figure 2. On the other hand, the diffusion selectivities of the former membranes were smaller than those of the latter membranes. This is due to the fact that with increasing GA or TEOS content, the degree of swelling of the membrane decreased as shown in Figure 9. The increase in the water permselectivity with increasing GA or TEOS content was mainly due to an increase in the solubility of water in the $\mathrm{CMCNa} / \mathrm{GA}$ cross-linked and $\mathrm{CMCNa} /$ TEOS hybrid membranes.

\subsection{TEM images of cross-linked and hybrid membranes}

Figure 11 shows TEM images of the $\mathrm{CMCNa}$ membrane, the $\mathrm{CMCNa} / \mathrm{GA}$ cross-linked membranes and $\mathrm{CMCNa}$ /TEOS hybrid membranes with 10 and $30 \mathrm{wt} \%$ of GA or TEOS content. The black portion in these images is dependent on the $\mathrm{Si}$ atom in TEOS. As can be seen from these TEM images, the structure of the CMCNa membrane was homogeneous but in the CMCNa/TEOS hybrid membranes, a phase separated structure was observed, especially in the hybrid membrane with $30 \mathrm{wt} \%$ of TEOS content which showed a heterogeneously dispersed cohesion of TEOS. On the other hand, in the $\mathrm{CMCNa} / \mathrm{GA}$ cross-linked membranes such a phase separated structure was not observed.

Based on the above results, the hypothetical structure of the $\mathrm{CMCNa}$ /TEOS hybrid membranes is shown in Figure 12. In the CMCNa/TEOS hybrid

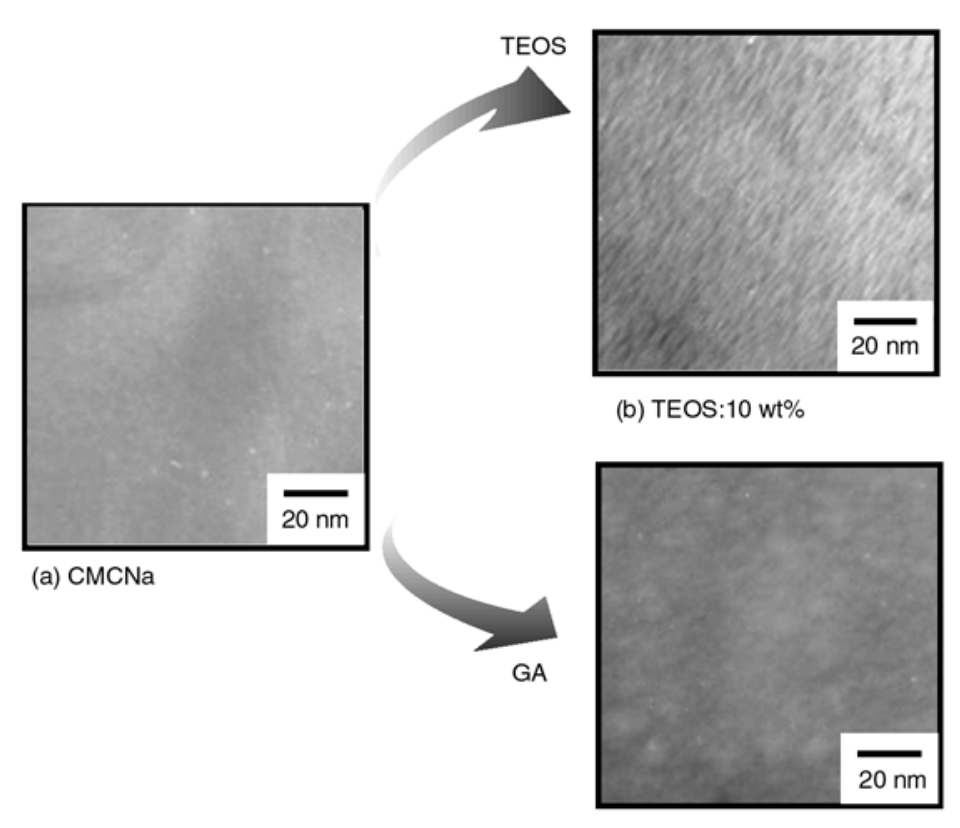

(d) GA:10 wt\%

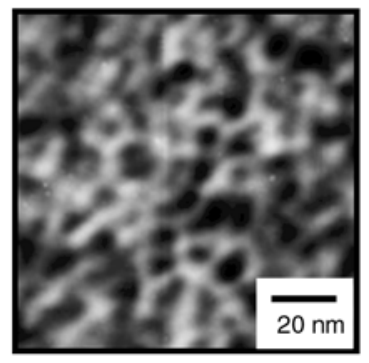

(c) TEOS:30 wt\%

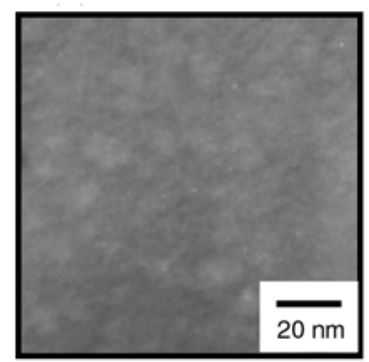

(e) GA:30 wt

Figure 11. TEM images of : (a) the CMCNa membrane, (b) with a TEOS content of $10 \mathrm{wt} \%$, (c) with a TEOS content of $30 \mathrm{wt} \%$ in CMCNa/TEOS hybrid membranes, (d) with a GA content of $10 \mathrm{wt} \%$, (e) with a GA content of $30 \mathrm{wt} \%$ in $\mathrm{CMCNa} / \mathrm{GA}$ cross-linked membrane

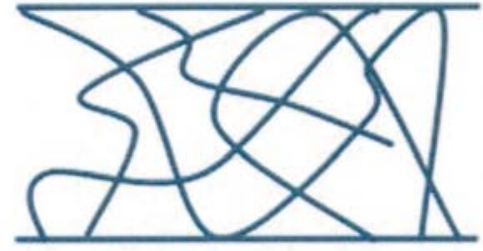

a) $\mathrm{CMCNa}$

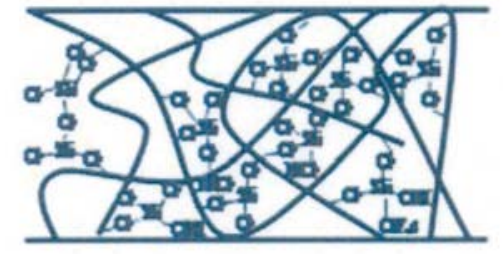

(b) $\mathrm{CMCNa} / \mathrm{TEOS}$ (TEOS: $10 \mathrm{wt} \%$ )

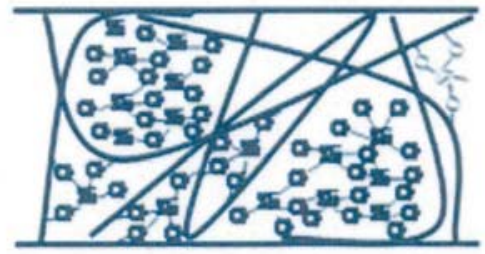

(c) $\mathrm{CMCNa}$ /TEOS (TEOS: $30 \mathrm{wt} \%$ )

Figure 12. Change in the cross-linked structure of CMCNa/TEOS hybrid membranes 
membranes containing $10 \mathrm{wt} \%$ TEOS or less, the cross-linked structure was formed by smooth reactions between the CMCNa and TEOS and between the TEOS molecules. Consequently, although the permeation rate decreased with increasing TEOS content, the water/ethanol selectivity was improved remarkably, as shown in Figure 1. However, in the $\mathrm{CMCNa} / \mathrm{TEOS}$ hybrid membrane containing excess TEOS $(>10 \mathrm{wt} \%)$ the polycondensation reaction between the TEOS molecules proceeds preferentially. Therefore, the cross-linking reaction between CMCNa and TEOS was less favored. The resulting cohesion in the TEOS domains prevents the hydrogen bonds between the CMCNa molecules. These results influence the diffusion path and consequently the permeation rates and the selectivity. Therefore, an increase in the permeation rate and a decrease in the water/ethanol selectivity were observed.

\section{Conclusions}

Both $\mathrm{CMCNa} / \mathrm{GA}$ cross-linked and $\mathrm{CMCNa} / \mathrm{TEOS}$ hybrid membranes showed high water/ethanol selectivity. Consequently, it was found that the introduction of the cross-linking structure into CMCNa molecule was very effective.The water/ ethanol selectivity of the $\mathrm{CMCNa}$ /TEOS hybrid membranes was higher than that of the $\mathrm{CMCNa} / \mathrm{GA}$ cross-linked membranes. When a suitable amount of TEOS was added to CMCNa, the TEOS was homogeneously dispersed in the membrane, and the most suitable cross-linked structure was formed. Consequently, the best water/ethanol selectivity was obtained. In comparing of the role of TEOS and GA as cross-linkers, since TEOS gave higher cross-link points than GA, a denser cross-linked structure was formed, the swelling of the membrane was effectively controlled. Consequently, the $\mathrm{CMCNa}$ /TEOS hybrid membranes showed higher water/ethanol selectivity. The abrupt changes in some properties of CMC membranes cross-linked with $10 \mathrm{wt} \%$ of TEOS can be explained as follows: in the sol-gel process the CMC membranes with $10 \mathrm{wt} \%$ of TEOS is homogeneous with a lot of hydroxyl groups and consequently chemical and physical structures of this membrane are very fine. In this study, we demonstrated that membranes with high water/ethanol selectivity can be designed by the hybridization of $\mathrm{CMCNa}$ as an organic component and TEOS as an inorganic component using the sol-gel reaction, and $\mathrm{CMCNa}$ offers potential for the selective separation of an azeotrope of ethanol/water.

\section{Acknowledgements}

This work was financially supported by the 'High-Tech Research Center' Project in Matching Fund Subsidy for Private Universities, 2005-2009.

\section{References}

[1] Baker R. W.: Pervaporation. in 'Membrane separation system, recent developments and future directions' (eds.: Baker R. W., Cusssler E. L., Eykamp W., Koros W. J., Riley R. L., Strathmann H.) Noyes Data Corporation, Park Ridge, USA, 151-187 (1991).

[2] Psaume R., Aptel Ph., Aurell Y., Mora C. J., Bersillon J. L.: Pervaporation: Importance of concentration polarization in the extraction of trace organics from water. Journal of Membrane Science, 36, 373-384 (1988). DOI: $10.1016 / 0376-7388(88) 80030-9$

[3] Blume I., Wijmans J. G., Baker R. W.: The separation of dissolved organics from water by pervaporation. Journal of Membrane Science, 49, 253-286 (1990). DOI: $\underline{10.1016 / \mathrm{S} 0376-7388(00) 80643-2}$

[4] Fang Y., Pham V. A., Matuura T., Santerre J. P., Narbaitz R. M.: Effect of surface-modifying macromolecules and solvent evaporation time on the performance of polyethersulfone membranes for the separation of chloroform/water mixtures by pervaporation. Journal of Applied Polymer Science, 54, 1937-1943 (1994).

DOI: 10.1002/app.1994.070541216

[5] Uragami T., Matsuda T., Okuno H., Miyata T.: Structure of chemically modified chitosan membranes and their characteristics of permeation and separation of aqueous ethanol solutions. Journal of Membrane Science, 88, 243-251 (1994). DOI: $10.1016 / 0376-7388(94) 87010-1$

[6] Uragami T., Tanaka Y., Nishida S.: Permeation and separation under high temperature and high pressure for ethanol/water vapors through cross-linked quaternized chitosan composite membranes. Desalination, 147, 449-454 (2002). DOI: $\underline{10.1016 / \mathrm{S} 0011-9164(02) 00642-2}$

[7] Uragami T., Takuno M., Miyata T.: Evapomeation characteristics of cross-linked quaternized chitosan membranes for the separation of an ethanol/water azeotrope. Macromolecular Chemistry and Physics, 203, 1162-1170 (2002).

DOI: $10.1002 / 1521-3935(200206) 203: 9<1162:: A I D-$ MACP1162>3.0.CO;2-Q 
[8] Uragami T., Yamamoto S., Miyata T.: Dehydration from alcohols by polyion complex cross-linked chitosan composite membranes during evapomeation. Biomacromolecules, 4, 137-144 (2003). DOI: $10.1021 / \mathrm{bm} 0256420$

[9] Uragami T., Okazaki K., Matsugi H., Miyata T.: Structure and permeation characteristics of an aqueous ethanol solution of organic-inorganic hybrid membranes composed of poly(vinyl alcohol) and tetraethoxysilane. Macromolecules, 35, 9156-9163 (2002). DOI: $10.1021 / \mathrm{ma} 020850 \mathrm{u}$

[10] Uragami T., Matsugi H., Miyata T.: Pervaporation characteristics of organic-inorganic hybrid membranes composed of poly(vinyl alcohol-c0-acrylic acid) and tetraethoxysilane for water/ethanol separation. Macromolecules, 38, 8440-8446 (2005).

DOI: $10.1021 / \mathrm{ma} 051450 \mathrm{k}$

[11] Uragami T., Katayama T., Miyata T.: Dehydration of an ethanol/water azeotrope by novel organic-inorganic hybrid membranes based on quaternized chitosan and tetraethoxysilane. Biomacromolecules, 5, 1567-1574 (2004).

DOI: $\underline{10.1021 / \mathrm{bm} 0498880}$

[12] Inui K., Miyata T., Uragami T.: Permeation and separation of organic liquid mixtures through liquid-crystalline polymer networks. Die Angewandte Makromolekulare Chemie, 240, 241-250 (1996).

DOI: $10.1002 / a p m c .1996 .052400123$
[13] Uragami T., Ohshima T., Miyata T.: Removal of benzene from an aqueous solution of dilute benzene by various cross-linked poly(dimethylsiloxane) membranes during pervaporation. Macromolecules, 36, 9430-9436 (2003).

DOI: $\underline{10.1021 / \mathrm{ma} 0345753}$

[14] Uragami T., Tsukamoto K., Miyata T.: Pervaporation characteristic $\mathrm{s}$ of a benzoylchiotsan membrane for benzene/ cychlohexane mixtures. Macromolecular Chemistry and Physics, 199, 49-54 (1998).

[15] Sakka S., Ito S., Kamiya K.: Electronic spectra of transition metal ions in gel-derived and melt-derived glasses. Journal of Non-Crystalline Solids, 71, 311315 (1985).

DOI: 10.1016/0022-3093(85)90301-1

[16] Binning R. C., Lee R. J., Jennings J. F., Martin E. C.: Separation of liquid mixtures by pervaporation. Industrial Engineering and Chemistry, 53, 45-50 (1961). DOI: $10.1021 /$ ie $50613 \mathrm{a} 030$

[17] Aptel P., Cuny J., Jozefonvicz J., Morel G., Neel J.: Liquid transport through membranes prepared by grafting of polar monomers onto poly(tetrafluoroethylene) films. III. Steady-state distribution in membrane during pervaporation. Journal of Applied Polymer Science, 18, 365-378 (1974). DOI: 10.1002/app.1974.070180205

[18] Uragami T., Meotoiwa T., Miyata T.: Effects of morphology of multicomponent polymer membranes containing calixarene on permselective removal of benzene from a dilute aqueous solution of benzene. Macromolecules, 36, 2041-2048 (2003).

DOI: $\underline{10.1021 / \mathrm{ma} 025863 \mathrm{~m}}$ 